\title{
Five Dimensional Interpolation in Practice
}

\author{
Daniel Trad (CGGVeritas)
}

\section{SUMMARY}

Computer power growth has made possible to extend interpolation algorithms to many dimensions, which has proven to be essential to deal with the increase of dimensions of seismic data, from two to three dimensions and from narrow to wide azimuth. Simultaneous interpolation in all five seismic data dimensions has the greatest chance of predicting missing data with correct amplitude and phase variations because well sampled dimensions help to constraint the more poorly sampled dimensions.

One method that can easily be extended to five dimensions is sparse Fourier reconstruction. The success of Fourier interpolation methods strongly depends on two factors; 1 ) having efficient Fourier transform operators that permit the use of large multidimensional data windows; and 2) constraining the spectrum along dimensions where seismic amplitudes change smoothly and where the band limited assumption remains valid.

This presentation will discuss two practical aspects of interpolation and regularization of land data:

1) The use of Fourier reconstruction by binning spatial coordinates. Non-orthogonality on the Fourier operator is replaced by non-orthogonality on the sampling operator, making possible the use of the fast Fourier transform that speeds up convergence.

2) Strategies for interpolation and regularization of land data regarding the design of the target geometry. 